\title{
Zwitterion-Dianion Complexes and Anion-Anion Clusters with Negative Dissociation Energies
}

\author{
Steven R. Kass \\ Department of Chemistry, University of Minnesota, Minneapolis, MN 55455 (USA) \\ email: kass@chem.umn.edu
}

Submitted to the

Journal of the American Chemical Society

$5 / 24 / 05$ 


\begin{tabular}{|c|c|c|c|c|c|}
\hline \multicolumn{6}{|c|}{ B3LYP/aug-cC-pvdz $=-377.154542$} \\
\hline 1 & 6 & 0 & -.784750 & .000007 & -.000025 \\
\hline 2 & 6 & 0 & .784749 & -.000011 & -.000025 \\
\hline 3 & 8 & 0 & 1.360886 & -.800501 & .802718 \\
\hline 4 & 8 & 0 & -1.360874 & .802892 & .800336 \\
\hline 5 & 8 & 0 & 1.360909 & .800486 & -.802706 \\
\hline 6 & 8 & 0 & -1.360921 & -.802874 & -.800311 \\
\hline
\end{tabular}

Oxalate Monoanion $\left(\mathrm{HO}_{2} \mathrm{CCO}_{2}{ }^{-}\right)$

B3LYP/aug-cC-pvdz $=-377.873326$

$\mathrm{zpe}=0.035912$, thermal correction $=0.041622$

\begin{tabular}{|c|c|c|c|c|c|}
\hline 1 & 6 & 0 & .000000 & .768571 & .000000 \\
\hline 2 & 6 & 0 & -.239270 & -.799877 & .000000 \\
\hline 3 & 8 & 0 & -1.399131 & -1.227631 & .000000 \\
\hline 4 & 8 & 0 & 1.328424 & 1.016013 & .000000 \\
\hline 5 & 8 & 0 & .874707 & -1.423025 & .000000 \\
\hline 6 & 8 & 0 & -.832689 & 1.649653 & .000000 \\
\hline 7 & 1 & 0 & 1.665135 & .067753 & .000000 \\
\hline
\end{tabular}

Oxalic Acid $\left(\mathrm{CO}_{2} \mathrm{H}\right)_{2}$

B3LYP/aug-cC-pvdz $=-378.395842$

zpe $=0.049302$, thermal correction $=0.055311$

$\begin{array}{rrrrrr}1 & 6 & 0 & -0.052377 & 0.770000 & 0.000000 \\ 2 & 6 & 0 & 0.052377 & -0.770000 & 0.000000 \\ 3 & 8 & 0 & 1.125766 & -1.326648 & 0.000000 \\ 4 & 8 & 0 & -1.125766 & -1.383519 & 0.000000 \\ 5 & 1 & 0 & -1.814772 & -0.689612 & 0.000000 \\ 6 & 8 & 0 & -1.125766 & 1.326648 & 0.000000 \\ 7 & 8 & 0 & 1.125766 & 1.383519 & 0.000000 \\ 8 & 1 & 0 & 1.814772 & 0.689612 & 0.000000\end{array}$

Glycine Zwitterion/Oxalate Dianion (1)

$\mathrm{B} 3 \mathrm{LYP} / \mathrm{aug}-\mathrm{cC}-\mathrm{pvdz}=-661.731376$

$\mathrm{zpe}=0.103427$, thermal correction $=0.113727$

$\begin{array}{rrrrrr}1 & 6 & 0 & 2.381854 & -.742257 & -.171635 \\ 2 & 6 & 0 & 2.289050 & .793593 & .146687 \\ 3 & 8 & 0 & 1.324471 & 1.163586 & .921931 \\ 4 & 1 & 0 & -.077263 & .447015 & .698962 \\ 5 & 7 & 0 & -.796639 & -.221306 & .258282 \\ 6 & 1 & 0 & -.066497 & -.779759 & -.301618 \\ 7 & 8 & 0 & 1.369440 & -1.267470 & -.777568 \\ 8 & 6 & 0 & -1.876802 & .417591 & -.528109 \\ 9 & 6 & 0 & -3.288506 & -.007688 & -.014857 \\ 10 & 8 & 0 & -3.299852 & -.802200 & .967578 \\ 11 & 8 & 0 & -4.257300 & .491285 & -.640270 \\ 12 & 1 & 0 & -1.783021 & .137605 & -1.584981 \\ 13 & 1 & 0 & -1.786748 & 1.509072 & -.463779 \\ 14 & 1 & 0 & -1.281079 & -.800359 & .957276 \\ 15 & 8 & 0 & 3.141601 & 1.552149 & -.359058 \\ 16 & 8 & 0 & 3.413828 & -1.353833 & .174094\end{array}$


HF/aug-cc-pvdz Geometry

$\begin{array}{lll}1 & 6 & 0 \\ 2 & 6 & 0 \\ 3 & 8 & 0 \\ 4 & 1 & 0 \\ 5 & 7 & 0 \\ 6 & 1 & 0 \\ 7 & 8 & 0 \\ 8 & 6 & 0 \\ 9 & 6 & 0 \\ 10 & 8 & 0 \\ 11 & 8 & 0 \\ 12 & 1 & 0 \\ 13 & 1 & 0 \\ 14 & 1 & 0 \\ 15 & 8 & 0 \\ 16 & 8 & 0\end{array}$

MP2 / aug-cc-pvdz Geometry

$\begin{array}{rrrrrr}1 & 6 & 0 & 2.370986 & -.726868 & -.183929 \\ 2 & 6 & 0 & 2.249487 & .793446 & .155030 \\ 3 & 8 & 0 & 1.305498 & 1.129078 & .986581 \\ 4 & 1 & 0 & -.070539 & .378340 & .760575 \\ 5 & 7 & 0 & -.785295 & -.270102 & .287681 \\ 6 & 1 & 0 & -.058595 & -.811497 & -.289072 \\ 7 & 8 & 0 & 1.366931 & -1.263574 & -.815042 \\ 8 & 6 & 0 & -1.833043 & .422262 & -.497809 \\ 9 & 6 & 0 & -3.257878 & -.001864 & -.027086 \\ 10 & 8 & 0 & -3.295878 & -.841872 & .930484 \\ 11 & 8 & 0 & -4.214492 & .540435 & -.654197 \\ 12 & 1 & 0 & -1.720334 & .178970 & -1.564201 \\ 13 & 1 & 0 & -1.723571 & 1.510224 & -.383725 \\ 14 & 1 & 0 & -1.302097 & -.866027 & .951112 \\ 15 & 8 & 0 & 3.066091 & 1.585167 & -.384157 \\ 16 & 8 & 0 & 3.421211 & -1.326879 & .165620\end{array}$

Deprotonated Glycine/Oxalate Monoanion (3)

B3LYP/aug-cC-pvdz $=-661.729787$

$\mathrm{zpe}=0.103162$, thermal correction $=0.113942$

$\begin{array}{rrrrrr}1 & 6 & 0 & -1.720084 & .113005 & -.148325 \\ 2 & 6 & 0 & -3.261482 & .088263 & .025175 \\ 3 & 8 & 0 & -3.688880 & .594320 & 1.095562 \\ 4 & 8 & 0 & -1.112205 & -.976027 & .345648 \\ 5 & 8 & 0 & -3.901590 & -.412068 & -.936330 \\ 6 & 8 & 0 & -1.110753 & 1.036145 & -.682953 \\ 7 & 1 & 0 & -.113677 & -.955799 & .223130 \\ 8 & 8 & 0 & 1.553561 & -1.409679 & .176378 \\ 9 & 6 & 0 & 2.585378 & -.685482 & .057707 \\ 10 & 8 & 0 & 3.749097 & -1.063674 & -.251872 \\ 11 & 6 & 0 & 2.399901 & .822687 & .375075 \\ 12 & 1 & 0 & 2.272476 & .899928 & 1.468492 \\ 13 & 1 & 0 & 1.460371 & 1.173523 & -.071701 \\ 14 & 7 & 0 & 3.540411 & 1.670986 & -.042957 \\ 15 & 1 & 0 & 4.310101 & 1.002504 & -.0159091 \\ 16 & 1 & 0 & 3.351723 & 1.999950 & -.989399\end{array}$


HF/aug-cc-pvdz Geometry

$\begin{array}{rrrrrr}1 & 6 & 0 & -1.855277 & .159026 & -.152297 \\ 2 & 6 & 0 & -3.383519 & .068607 & .036680 \\ 3 & 8 & 0 & -3.800510 & .512198 & 1.109641 \\ 4 & 8 & 0 & -1.212349 & -.904483 & .291505 \\ 5 & 8 & 0 & -3.996147 & -.422824 & -.914623 \\ 6 & 8 & 0 & -1.300184 & 1.100961 & -.651474 \\ 7 & 1 & 0 & -.256807 & -.842885 & .169224 \\ 8 & 8 & 0 & 1.604075 & -1.292284 & .160470 \\ 9 & 6 & 0 & 2.683341 & -.689032 & .057597 \\ 10 & 8 & 0 & 3.795654 & -1.160618 & -.220295 \\ 11 & 6 & 0 & 2.627855 & .822135 & .363295 \\ 12 & 1 & 0 & 2.526106 & .913486 & 1.448241 \\ 13 & 1 & 0 & 1.719993 & 1.240162 & -.066441 \\ 14 & 7 & 0 & 3.797266 & 1.590397 & -.072571 \\ 15 & 1 & 0 & 4.573911 & .952921 & -.021576 \\ 16 & 1 & 0 & 3.697228 & 1.775532 & -1.054892\end{array}$

MP2 / aug-cc-pvdz Geometry

$\begin{array}{rrrrrr}1 & 6 & 0 & -1.643496 & .038948 & -.203857 \\ 2 & 6 & 0 & -3.165360 & .117604 & .048474 \\ 3 & 8 & 0 & -3.513563 & .839869 & 1.032808 \\ 4 & 8 & 0 & -1.050597 & -.971086 & .466004 \\ 5 & 8 & 0 & -3.881730 & -.532326 & -.774518 \\ 6 & 8 & 0 & -1.026341 & .817886 & -.941551 \\ 7 & 1 & 0 & -.059500 & -1.010857 & .275824 \\ 8 & 8 & 0 & 1.568518 & -1.485110 & .185611 \\ 9 & 6 & 0 & 2.544897 & -.671139 & .069908 \\ 10 & 8 & 0 & 3.747068 & -.948376 & -.037771 \\ 11 & 6 & 0 & 2.226174 & .811905 & .392152 \\ 12 & 1 & 0 & 2.104663 & .877459 & 1.488543 \\ 13 & 1 & 0 & 1.255674 & 1.076833 & -.055108 \\ 14 & 7 & 0 & 3.286034 & 1.760031 & -.036361 \\ 15 & 1 & 0 & 4.110306 & 1.152868 & -.0142304 \\ 16 & 1 & 0 & 3.066483 & 2.032712 & -.997166\end{array}$

Oxalate Monoanion Dimer $\left(\mathrm{HO}_{2} \mathrm{CCO}_{2}^{-}\right)$

B3LYP/aug-cC-pvdz $=-755.658866$

$\mathrm{zpe}=0.071791$

thermal correction $=0.082463$

$$
\begin{array}{r}
1.564234 \\
2.437973 \\
3.620186 \\
1.836896 \\
0.239204 \\
-0.655265 \\
-1.564682 \\
-2.437674 \\
-3.619600 \\
-1.836345 \\
-0.239233 \\
0.656113 \\
-1.679544 \\
1.677673
\end{array}
$$

0.008485
0.018528
0.410023
-0.413925
-0.720210
-0.992248
-0.008724
-0.018814
-0.411462
0.415099
0.722166
0.993328
0.833198
-0.833864 
MP2 / aug-cc-pvdz Geometry

$\begin{array}{rrrrrr}1 & 6 & 0 & 1.490830 & -0.861685 & 0.007915 \\ 2 & 6 & 0 & 2.423673 & 0.368543 & 0.015888 \\ 3 & 8 & 0 & 3.611017 & 0.204719 & 0.405443 \\ 4 & 8 & 0 & 1.868122 & 1.443406 & -0.414699 \\ 5 & 1 & 0 & 0.284055 & 1.262034 & -0.747755 \\ 6 & 8 & 0 & -0.602194 & 0.834316 & -1.021575 \\ 7 & 6 & 0 & -1.490910 & 0.861739 & -0.007921 \\ 8 & 6 & 0 & -2.423627 & -0.368586 & -0.015888 \\ 9 & 8 & 0 & -3.610978 & -0.204911 & -0.405457 \\ 10 & 8 & 0 & -1.867933 & -1.443387 & 0.414688 \\ 11 & 1 & 0 & -0.284221 & -1.261709 & 0.747810 \\ 12 & 8 & 0 & 0.602217 & -0.834297 & 1.021665 \\ 13 & 8 & 0 & -1.541654 & 1.741619 & 0.853534 \\ 14 & 8 & 0 & 1.541450 & -1.741512 & -0.853603\end{array}$

Oxalate Monoanion Trimer $\left(\mathrm{HO}_{2} \mathrm{CCO}_{2}^{-}\right)_{3}$

B3LYP/aug-cC-pvdz $=-1133.394423$

$\mathrm{zpe}=0.109074$, thermal correction $=0.124662$

$\begin{array}{rrrrrr}1 & 6 & 0 & -2.448391 & -1.577574 & 0.293335 \\ 2 & 6 & 0 & -3.668010 & -0.732229 & -0.164306 \\ 3 & 8 & 0 & -4.705807 & -1.363600 & -0.483800 \\ 4 & 8 & 0 & -3.492116 & 0.521247 & -0.132786 \\ 5 & 1 & 0 & -1.951626 & 1.389363 & -0.224833 \\ 6 & 8 & 0 & -2.432118 & -2.214961 & 1.340664 \\ 7 & 8 & 0 & -1.435474 & -1.508400 & -0.570314 \\ 8 & 1 & 0 & -0.564426 & -1.870798 & -0.227093 \\ 9 & 8 & 0 & -1.125339 & 1.823005 & -0.592475 \\ 10 & 8 & 0 & 1.101195 & -2.096792 & 0.152303 \\ 11 & 6 & 0 & 0.945726 & 2.991236 & -0.259756 \\ 12 & 6 & 0 & -0.401816 & 2.485478 & 0.312405 \\ 13 & 8 & 0 & -0.756609 & 2.690876 & 1.468695 \\ 14 & 6 & 0 & 3.322338 & -1.293712 & 0.349175 \\ 15 & 6 & 0 & 2.176932 & -1.923861 & -0.488224 \\ 16 & 8 & 0 & 2.472143 & -2.227597 & -1.665520 \\ 17 & 8 & 0.956276 & 4.187208 & -0.649712 \\ 18 & 8 & 1.901161 & 2.165318 & -0.250374 \\ 19 & 8 & 0 & 0.225612 & -1.978410 & 0.825484 \\ 20 & 8 & 0 & 0.336796 & 0.029130 & 0.559532 \\ 21 & 1 & 0 & 2.589629 & 0.569216 & 0.182567\end{array}$

Malonate Dianion $\mathrm{CH}_{2}\left(\mathrm{CO}_{2}^{-}\right)_{2}$

B3LYP/aug-cC-pvdz $=-416.488592$

$\mathrm{zpe}=0.050320$, thermal correction $=0.056907$

$\begin{array}{rrrrrr}1 & 6 & 0 & -.001154 & -.012944 & .786839 \\ 2 & 6 & 0 & -1.353128 & .103623 & .009322 \\ 3 & 8 & 0 & -2.257053 & -.737987 & .318580 \\ 4 & 8 & 0 & -1.473803 & 1.066467 & -.801359 \\ 5 & 8 & 0 & 2.247538 & .743506 & .330499 \\ 6 & 6 & 0 & 1.353091 & -.103955 & .010644 \\ 7 & 8 & 0 & 1.484945 & -1.056233 & -.811235 \\ 8 & 1 & 0 & .060411 & .867497 & 1.448036 \\ 9 & 1 & 0 & -.066279 & -.913864 & 1.419267\end{array}$


Malonate Monoanion $\mathrm{HO}_{2} \mathrm{CCH}_{2} \mathrm{CO}_{2}^{-}$

B3LYP/aug-cC-pvdz $=-417.206420$

$\mathrm{zpe}=0.062132$

thermal correction $=0.068704$

$\begin{array}{lrrrrr}1 & 6 & 0 & -.010196 & -.877855 & .007081 \\ 2 & 6 & 0 & -1.334428 & -.068263 & -.000205 \\ 3 & 8 & 0 & -1.212930 & 1.214697 & -.000313 \\ 4 & 8 & 0 & -2.396441 & -.708458 & -.004457 \\ 5 & 8 & 0 & 1.219437 & 1.203719 & .001138 \\ 6 & 6 & 0 & 1.319274 & -.111057 & .000004 \\ 7 & 8 & 0 & 2.393497 & -.705505 & -.005264 \\ 8 & 1 & 0 & -.012989 & -1.535134 & .888152 \\ 9 & 1 & 0 & -.013155 & -1.553789 & -.859686 \\ 10 & 1 & 0 & .149740 & 1.396353 & .001417\end{array}$

Malonic acid $\mathrm{CH}_{2}\left(\mathrm{CO}_{2} \mathrm{H}\right)_{2}$

B3LYP/aug-cC-pvdz $=-417.723984$

zpe $=0.077153,($ Has 1 imaginary frequency at -15.8$)$

$\begin{array}{rrrrrr}1 & 6 & 0 & 0.000000 & 0.837977 & 0.000000 \\ 2 & 6 & 0 & -1.224887 & -0.043029 & 0.000000 \\ 3 & 8 & 0 & -1.227879 & -1.262782 & 0.000000 \\ 4 & 8 & 0 & -2.360455 & 0.676066 & 0.000000 \\ 5 & 1 & 0 & -3.107588 & 0.053678 & 0.000000 \\ 6 & 6 & 0 & 1.380554 & 0.166357 & 0.000000 \\ 7 & 1 & 0 & -0.047798 & 1.504406 & 0.872686 \\ 8 & 1 & 0 & -0.047798 & 1.504406 & -0.872686 \\ 9 & 8 & 0 & 2.377910 & 0.846689 & 0.000000 \\ 10 & 8 & 0 & 1.429750 & -1.171958 & 0.000000 \\ 11 & 1 & 0 & 0.514571 & -1.534435 & 0.000000\end{array}$

Glycine Zwitterion/Malonate Dianion (2)

$\mathrm{B} 3 \mathrm{LYP} / \mathrm{aug}-\mathrm{CC}-\mathrm{pvdz}=-701.063890$

$\mathrm{zpe}=0.131892$, thermal correction $=0.142949$

$\begin{array}{rrrrrr}1 & 6 & 0 & -2.311758 & .000210 & .804115 \\ 2 & 6 & 0 & -2.141886 & 1.334913 & .011300 \\ 3 & 8 & 0 & -3.183650 & 1.945546 & -.313727 \\ 4 & 8 & 0 & -.944949 & 1.742005 & -.212472 \\ 5 & 1 & 0 & .301974 & .818030 & .087036 \\ 6 & 7 & 0 & .991788 & -.000706 & .209246 \\ 7 & 1 & 0 & .302376 & -.819519 & .086484 \\ 8 & 8 & 0 & -.945897 & -1.741890 & -.213481 \\ 9 & 6 & 0 & -2.142553 & -1.335039 & .012001 \\ 10 & 8 & 0 & -3.184763 & -1.944671 & -.313600 \\ 11 & 6 & 0 & 2.138303 & -.000141 & -.730748 \\ 12 & 6 & 0 & 3.500499 & -.000066 & .032117 \\ 13 & 8 & 0 & 3.422326 & -.000348 & 1.293733 \\ 14 & 8 & 0 & 4.523121 & .000278 & -.0697133 \\ 15 & 1 & 0 & 2.089423 & .886593 & -1.374748 \\ 16 & 1 & 0 & 2.089723 & -.886514 & -1.375267 \\ 17 & 1 & 0 & 1.429768 & -.000868 & 1.141734 \\ 18 & 1 & 0 & -1.577241 & .000184 & 1.626662 \\ 19 & 1 & 0 & -3.323689 & .000421 & 1.224103\end{array}$


HF/aug-cc-pvdz Geometry

$\begin{array}{rrrrrr}1 & 6 & 0 & -2.440443 & .000028 & .775423 \\ 2 & 6 & 0 & -2.178744 & 1.322046 & .009565 \\ 3 & 8 & 0 & -3.162633 & 1.934371 & -.399767 \\ 4 & 8 & 0 & -.983018 & 1.698582 & -.096315 \\ 5 & 1 & 0 & .398477 & .792945 & .180626 \\ 6 & 7 & 0 & 1.057969 & -.000054 & .279657 \\ 7 & 1 & 0 & .398438 & -.792956 & .180195 \\ 8 & 8 & 0 & -.983265 & -1.698253 & -.097247 \\ 9 & 6 & 0 & -2.178992 & -1.321946 & .009397 \\ 10 & 8 & 0 & -3.163031 & -1.934467 & -.399281 \\ 11 & 6 & 0 & 2.164110 & .000180 & -.697289 \\ 12 & 6 & 0 & 3.545276 & -.000110 & -.000158 \\ 13 & 8 & 0 & 3.525771 & -.000470 & 1.238936 \\ 14 & 8 & 0 & 4.509212 & .000082 & -.768095 \\ 15 & 1 & 0 & 2.086476 & .879270 & -1.330322 \\ 16 & 1 & 0 & 2.086372 & -.878507 & -1.330868 \\ 17 & 1 & 0 & 1.492944 & -.000317 & 1.190950 \\ 18 & 1 & 0 & -1.798221 & -.000108 & 1.660922 \\ 19 & 1 & 0 & -3.481802 & .000101 & 1.083419\end{array}$

MP2 / aug-cc-pvdz Geometry

$\begin{array}{rrr}-2.205119 & -.000110 & .840749 \\ -2.112051 & 1.318758 & .015696 \\ -3.193661 & 1.875351 & -.311338 \\ -.926018 & 1.767279 & -.244978 \\ .281611 & .825912 & .113015 \\ .962230 & .000122 & .239277 \\ .281613 & -.825624 & .112576 \\ -.925891 & -1.766927 & -.245782 \\ -2.111962 & -1.318903 & .015585 \\ -3.193527 & -1.875546 & -.311511 \\ 2.090688 & .000381 & -.722689 \\ 3.459419 & -.000112 & .023963 \\ 3.392432 & -.000850 & 1.297044 \\ 4.480750 & .000420 & -.723274 \\ 2.030837 & . .891228 & -1.364386 \\ 2.030621 & -.889856 & -1.365201 \\ 1.422954 & -.000128 & 1.163114 \\ -1.400326 & -.000126 & 1.598357 \\ -3.181456 & -.000173 & 1.346474\end{array}$


Deprotonated Glycine/Malonate Monoanion (4)

B3LYP/aug-cC-pvdz $=-701.057384$

$\mathrm{zpe}=0.131224$, thermal correction $=0.142748$

$\begin{array}{rrrrrr}1 & 6 & 0 & -1.066704 & -.167720 & .519654 \\ 2 & 8 & 0 & -.406250 & -1.016816 & -.271601 \\ 3 & 8 & 0 & -.534089 & .801000 & 1.059389 \\ 4 & 1 & 0 & .580028 & -.814421 & -.335118 \\ 5 & 8 & 0 & 2.220430 & -1.005135 & -.571558 \\ 6 & 6 & 0 & 3.308507 & -.512534 & -.141028 \\ 7 & 8 & 0 & 4.392732 & -1.122999 & .044658 \\ 8 & 6 & 0 & 3.337591 & 1.010294 & .187374 \\ 9 & 1 & 0 & 3.480740 & 1.102791 & 1.276194 \\ 10 & 1 & 0 & 4.257216 & 1.410663 & -.271142 \\ 11 & 7 & 0 & 2.195455 & 1.840982 & -.204742 \\ 12 & 1 & 0 & 1.959013 & 1.615203 & -1.170579 \\ 13 & 1 & 0 & 1.364092 & 1.548364 & .316518 \\ 14 & 6 & 0 & -2.511798 & -.547784 & .735956 \\ 15 & 1 & 0 & -2.613855 & -1.640068 & .683463 \\ 16 & 1 & 0 & -2.797662 & -.217814 & 1.743194 \\ 17 & 6 & 0 & -3.597567 & .051493 & -.253780 \\ 18 & 8 & 0 & -3.199211 & .772839 & -1.195332 \\ 19 & 8 & 0 & -4.775853 & -.290651 & .047143\end{array}$

HF/aug-cc-pvdz Geometry

$\begin{array}{rrrrrr}1 & 6 & 0 & -1.148487 & -.120863 & .530186 \\ 2 & 8 & 0 & -.511100 & -.965925 & -.251696 \\ 3 & 8 & 0 & -.608332 & .813249 & 1.061797 \\ 4 & 1 & 0 & .434414 & -.775467 & -.317600 \\ 5 & 8 & 0 & 2.243696 & -.947663 & -.557321 \\ 6 & 6 & 0 & 3.347212 & -.550918 & -.147056 \\ 7 & 8 & 0 & 4.364910 & -1.223860 & .060514 \\ 8 & 6 & 0 & 3.503745 & .959429 & .137098 \\ 9 & 1 & 0 & 3.745600 & 1.061824 & 1.197386 \\ 10 & 1 & 0 & 4.392908 & 1.289899 & -.405812 \\ 11 & 7 & 0 & 2.390994 & 1.839788 & -.180780 \\ 12 & 1 & 0 & 2.090861 & 1.643656 & -1.118836 \\ 13 & 1 & 0 & 1.593421 & 1.591805 & .379428 \\ 14 & 6 & 0 & -2.593129 & -.489353 & .761567 \\ 15 & 1 & 0 & -2.685964 & -1.574195 & .779650 \\ 16 & 1 & 0 & -2.880228 & -.102627 & 1.737507 \\ 17 & 6 & 0 & -3.636780 & .046041 & -.265866 \\ 18 & 8 & 0 & -3.224327 & .752463 & -1.184182 \\ 19 & 8 & 0 & -4.797765 & -.313192 & -.014341\end{array}$


MP2 / aug-cc-pvdz Geometry

$\begin{array}{rrrrrr}1 & 6 & 0 & -1.038517 & -.315381 & .520597 \\ 2 & 8 & 0 & -.392120 & -.999195 & -.436928 \\ 3 & 8 & 0 & -.488843 & .517033 & 1.254764 \\ 4 & 1 & 0 & .599274 & -.800834 & -.436927 \\ 5 & 8 & 0 & 2.227837 & -.986748 & -.656347 \\ 6 & 6 & 0 & 3.275286 & -.446140 & -.158806 \\ 7 & 8 & 0 & 4.393621 & -1.001705 & .057466 \\ 8 & 6 & 0 & 3.200223 & 1.062429 & .210691 \\ 9 & 1 & 0 & 3.339457 & 1.137914 & 1.303337 \\ 10 & 1 & 0 & 4.088388 & 1.538157 & -.243581 \\ 11 & 7 & 0 & 1.995634 & 1.817123 & -.161865 \\ 12 & 1 & 0 & 1.756564 & 1.551335 & -1.120494 \\ 13 & 1 & 0 & 1.204154 & 1.454280 & .382363 \\ 14 & 6 & 0 & -2.492640 & -.703831 & .644107 \\ 15 & 1 & 0 & -2.617825 & -1.764062 & .374486 \\ 16 & 1 & 0 & -2.795661 & -.566004 & 1.693460 \\ 17 & 6 & 0 & -3.510328 & .117918 & -.236348 \\ 18 & 8 & 0 & -3.036978 & 1.014059 & -.989293 \\ 19 & 8 & 0 & -4.722009 & -.238520 & -.067291\end{array}$

Glycine Zwitterion/Malonate Dianion Minus An Electron

B3LYP/aug-cc-pvdz $=-701.034296$ (-701.017874 at anion geometry) $\mathrm{zpe}=0.130661$, thermal correction $=0.142302$

$\begin{array}{rrrrrr}1 & 6 & 0 & 3.042045 & .096574 & -.076864 \\ 2 & 6 & 0 & 2.254683 & -1.264040 & -.252198 \\ 3 & 8 & 0 & 2.962348 & -2.170407 & .252613 \\ 4 & 8 & 0 & 1.180745 & -1.303509 & -.864698 \\ 5 & 1 & 0 & -.503957 & -.815909 & -.273409 \\ 6 & 7 & 0 & -1.151493 & -.201710 & .243309 \\ 7 & 1 & 0 & -.518900 & .465693 & .710254 \\ 8 & 8 & 0 & 1.143365 & 1.214394 & 1.014138 \\ 9 & 6 & 0 & 2.099451 & 1.326948 & .237258 \\ 10 & 8 & 0 & 2.573943 & 2.322228 & -.363954 \\ 11 & 6 & 0 & -2.215555 & .429506 & -.594095 \\ 12 & 6 & 0 & -3.593561 & -.034336 & -.010264 \\ 13 & 8 & 0 & -3.479467 & -.801613 & .995082 \\ 14 & 8 & 0 & -4.604147 & .395534 & -.589304 \\ 15 & 1 & 0 & -2.114591 & .106916 & -1.635156 \\ 16 & 1 & 0 & -2.129959 & 1.519822 & -.549993 \\ 17 & 1 & 0 & -1.762814 & -.733888 & .927156 \\ 18 & 1 & 0 & 3.756084 & .036818 & .753623 \\ 19 & 1 & 0 & 3.597905 & .251586 & -1.009664\end{array}$


Deprotonated Glycine/Malonate Monoanion Minus An Electron

B3LYP/aug-cC-pvdz $=-701.023395$ (-701.014751 at anion geometry) $\mathrm{zpe}=0.129403$, thermal correction $=0.141219$

$\begin{array}{rrrrrr}1 & 6 & 0 & 1.173945 & -.067297 & -.736847 \\ 2 & 8 & 0 & .637718 & -1.216571 & -.344710 \\ 3 & 8 & 0 & .541955 & .944046 & -1.000377 \\ 4 & 1 & 0 & -.363722 & -1.158562 & -.248611 \\ 5 & 8 & 0 & -1.955693 & -1.439882 & .009210 \\ 6 & 6 & 0 & -2.945806 & -.680008 & .194523 \\ 7 & 8 & 0 & -4.028778 & -.892231 & .756830 \\ 8 & 6 & 0 & -2.780794 & .771437 & -.469982 \\ 9 & 1 & 0 & -3.185603 & .629481 & -1.484941 \\ 10 & 1 & 0 & -1.718477 & 1.023634 & -.545533 \\ 11 & 7 & 0 & -3.517100 & 1.800883 & .215117 \\ 12 & 1 & 0 & -4.418661 & 1.436869 & .520497 \\ 13 & 1 & 0 & -3.017566 & 2.133666 & 1.035559 \\ 14 & 6 & 0 & 2.688510 & -.145605 & -.857200 \\ 15 & 1 & 0 & 2.987396 & -1.149185 & -1.186071 \\ 16 & 1 & 0 & 3.014707 & .587234 & -1.605258 \\ 17 & 6 & 0 & 3.443188 & .157489 & .440427 \\ 18 & 8 & 0 & 2.835844 & .524630 & 1.476439 \\ 19 & 8 & 0 & 4.699875 & .039329 & .425485\end{array}$

Malonate monoanion/ $\mathrm{Cl}^{-}$

$\mathrm{B} 3 \mathrm{LYP} / \mathrm{aug}-\mathrm{CC}-\mathrm{pvdz}=-877.416999$

$\mathrm{zpe}=0.063834$, thermal correction $=0.071955$

$\begin{array}{lrrrrr}1 & 6 & 0 & .144225 & .602484 & -.052957 \\ 2 & 8 & 0 & -.754539 & -.330145 & -.424618 \\ 3 & 8 & 0 & -.120716 & 1.526705 & .702380 \\ 4 & 1 & 0 & -1.660876 & -.153292 & -.065559 \\ 5 & 17 & 0 & -4.051882 & -.331885 & -.021872 \\ 6 & 6 & 0 & 1.485465 & .418804 & -.726473 \\ 7 & 1 & 0 & 1.352749 & -.130715 & -1.667970 \\ 8 & 1 & 0 & 1.875126 & 1.418495 & -.960595 \\ 9 & 6 & 0 & 2.635159 & -.326261 & .071551 \\ 10 & 8 & 0 & 2.349623 & -.838807 & 1.176670 \\ 11 & 8 & 0 & 3.741369 & -.315578 & -.540280\end{array}$

HF/aug-cc-pvdz Geometry

$\begin{array}{rrrrrr}1 & 6 & 0 & -.457128 & 1.189444 & -.175839 \\ 2 & 8 & 0 & .721801 & 1.316582 & .421095 \\ 3 & 8 & 0 & -1.136278 & 2.164545 & -.327741 \\ 4 & 1 & 0 & 1.277446 & .544229 & .366539 \\ 5 & 17 & 0 & 3.654317 & -.661324 & -.013118 \\ 6 & 6 & 0 & -.863820 & -.175051 & -.684276 \\ 7 & 1 & 0 & -1.177045 & -.056078 & -1.719026 \\ 8 & 1 & 0 & -.021546 & -.867049 & -.658044 \\ 9 & 6 & 0 & -2.033570 & -.834838 & .109865 \\ 10 & 8 & 0 & -2.107226 & -.560926 & 1.309332 \\ 11 & 8 & 0 & -2.737690 & -1.602191 & -.560805\end{array}$


MP2 / aug-cc-pvdz Geometry

$\begin{array}{lrrrrr}1 & 6 & 0 & .140153 & .586853 & -.052066 \\ 2 & 8 & 0 & -.755003 & -.340469 & -.468520 \\ 3 & 8 & 0 & -.125571 & 1.480362 & .752016 \\ 4 & 1 & 0 & -1.656619 & -.171179 & -.089336 \\ 5 & 17 & 0 & -4.005999 & -.314638 & -.022520 \\ 6 & 6 & 0 & 1.480818 & .436401 & -.736573 \\ 7 & 1 & 0 & 1.355878 & -.096775 & -1.691746 \\ 8 & 1 & 0 & 1.865143 & 1.446929 & -.946365 \\ 9 & 6 & 0 & 2.607519 & -.316239 & .067581 \\ 10 & 8 & 0 & 2.282374 & -.867296 & 1.156043 \\ 11 & 8 & 0 & 3.744030 & -.281624 & -.509961\end{array}$

Malonate Monoanion/ $\mathrm{Cl}^{-}$Minus An Electron [structure derived from dianion] B3LYP/aug-cC-pvdz $=-877.388005$ (-877.377585 at anion geometry) $\mathrm{zpe}=0.062210$, thermal correction $=0.070420$

$\begin{array}{rrrrrr}1 & 6 & 0 & .034755 & .727804 & -.071953 \\ 2 & 8 & 0 & -.674989 & -.263086 & -.590343 \\ 3 & 8 & 0 & -.371181 & 1.581366 & .693207 \\ 4 & 1 & 0 & -1.641796 & -.249933 & -.292508 \\ 5 & 17 & 0 & -3.616398 & -.452939 & -.005477 \\ 6 & 6 & 0 & 1.475569 & .716023 & -.593967 \\ 7 & 1 & 0 & 1.473421 & .517088 & -1.674011 \\ 8 & 1 & 0 & 1.912411 & 1.706321 & -.414251 \\ 9 & 6 & 0 & 2.383472 & -.307210 & .059332 \\ 10 & 8 & 0 & 2.109247 & -.858795 & 1.154465 \\ 11 & 8 & 0 & 3.483418 & -.596137 & -.0493153\end{array}$

$1,2-\mathrm{C}_{6} \mathrm{H}_{4}\left(\mathrm{CO}_{2}^{-}\right)_{2}$

B3LYP/aug-cC-pvdz $=-608.266160$

$\mathrm{zpe}=0.101884$

thermal correction $=0.111372$

$\begin{array}{rrrrrr}1 & 1 & 0 & -1.383069 & -2.472395 & .034101 \\ 2 & 6 & 0 & -1.404177 & -1.380629 & .030732 \\ 3 & 6 & 0 & -2.628176 & -.704507 & .016643 \\ 4 & 6 & 0 & -2.630383 & .696282 & -.016924 \\ 5 & 6 & 0 & -1.408511 & 1.376251 & -.030871 \\ 6 & 6 & 0 & -.166804 & .709283 & -.020115 \\ 7 & 6 & 0 & -.164569 & -.709754 & .020060 \\ 8 & 6 & 0 & 1.076970 & -1.625045 & .139095 \\ 9 & 8 & 0 & 1.141352 & -2.563470 & -.708216 \\ 10 & 8 & 0 & 1.829691 & -1.406881 & 1.121582 \\ 11 & 6 & 0 & 1.071922 & 1.628422 & -.138991 \\ 12 & 8 & 0 & 1.826153 & 1.411822 & -1.120652 \\ 13 & 8 & 0 & 1.132673 & 2.567741 & .707622 \\ 14 & 1 & 0 & -1.390799 & 2.468075 & -.034184 \\ 15 & 1 & 0 & -3.573338 & 1.253430 & -.029627 \\ 16 & 1 & 0 & -3.569373 & -1.264623 & .029253\end{array}$


B3LYP/aug-cC-pvdz $=-608.967529$

$\mathrm{zpe}=0.113092$

thermal correction $=0.122504$

$\begin{array}{rrrrrr}1 & 1 & 0 & 2.029979 & -1.987098 & 0.000000 \\ 2 & 6 & 0 & 1.766221 & -0.931312 & 0.000000 \\ 3 & 6 & 0 & 2.750119 & 0.052646 & 0.000000 \\ 4 & 6 & 0 & 2.368953 & 1.397814 & 0.000000 \\ 5 & 6 & 0 & 1.015145 & 1.719379 & 0.000000 \\ 6 & 6 & 0 & 0.000000 & 0.740936 & 0.000000 \\ 7 & 6 & 0 & 0.388706 & -0.630795 & 0.000000 \\ 8 & 6 & 0 & -0.506215 & -1.892178 & 0.000000 \\ 9 & 8 & 0 & 0.049702 & -2.996302 & 0.000000 \\ 10 & 8 & 0 & -1.790579 & -1.743488 & 0.000000 \\ 11 & 1 & 0 & -2.123988 & -0.601964 & 0.000000 \\ 12 & 6 & 0 & -1.423542 & 1.345389 & 0.000000 \\ 13 & 8 & 0 & -2.438990 & 0.545121 & 0.000000 \\ 14 & 8 & 0 & -1.529298 & 2.577040 & 0.000000 \\ 15 & 1 & 0 & 0.685875 & 2.756604 & 0.000000 \\ 16 & 1 & 0 & 3.119770 & 2.190900 & 0.000000 \\ 17 & 1 & 0 & 3.805373 & -0.228677 & 0.000000\end{array}$

$1,2-\mathrm{HO}_{2} \mathrm{CC}_{6} \mathrm{H}_{4} \mathrm{CO}_{2}^{-} / \mathrm{Cl}^{-}$

B3LYP/aug-cC-pvdz $=-1069.178917$

$\mathrm{zpe}=0.115439$

thermal correction $=0.126368$

$\begin{array}{rrrrrr}1 & 1 & 0 & -0.602234 & 2.332282 & -0.088491 \\ 2 & 6 & 0 & 0.385580 & 1.875038 & -0.053236 \\ 3 & 6 & 0 & 1.528752 & 2.675878 & -0.023477 \\ 4 & 6 & 0 & 2.787300 & 2.062399 & 0.016520 \\ 5 & 6 & 0 & 2.876678 & 0.670105 & 0.025383 \\ 6 & 6 & 0 & 1.736710 & -0.158209 & 0.007455 \\ 7 & 6 & 0 & 0.469110 & 0.469495 & -0.043974 \\ 8 & 6 & 0 & -0.825379 & -0.298188 & -0.192131 \\ 9 & 8 & 0 & -0.931503 & -1.325160 & -0.833650 \\ 10 & 8 & 0 & -1.843949 & 0.345356 & 0.403026 \\ 11 & 1 & 0 & -2.750307 & -0.020088 & 0.185215 \\ 12 & 17 & 0 & -4.926625 & 0.002521 & 0.027601 \\ 13 & 6 & 0 & 2.013376 & -1.671495 & 0.146879 \\ 14 & 8 & 0 & 2.725517 & -2.170428 & -0.764390 \\ 15 & 8 & 0 & 1.585561 & -2.190150 & 1.206511 \\ 16 & 1 & 0 & 3.853018 & 0.183387 & 0.038134 \\ 17 & 1 & 0 & 3.697164 & 2.669764 & 0.038291 \\ 18 & 1 & 0 & 1.437207 & 3.764704 & -0.034867\end{array}$


$1,4-\mathrm{C}_{6} \mathrm{H}_{4}\left(\mathrm{CO}_{2}^{-}\right)_{2}$

$\mathrm{B} 3 \mathrm{LYP} / \mathrm{aug}-\mathrm{cC}-\mathrm{pvdz}=-608.308123$

$\mathrm{zpe}=0.103322$

thermal correction $=0.112659$

$\begin{array}{rrrrrr}1 & 1 & 0 & -1.383069 & -2.472395 & .034101 \\ 2 & 6 & 0 & -1.404177 & -1.380629 & .030732 \\ 3 & 6 & 0 & -2.628176 & -.704507 & .016643 \\ 4 & 6 & 0 & -2.630383 & .696282 & -.016924 \\ 5 & 6 & 0 & -1.408511 & 1.376251 & -.030871 \\ 6 & 6 & 0 & -.166804 & .709283 & -.020115 \\ 7 & 6 & 0 & -.164569 & -.709754 & .020060 \\ 8 & 6 & 0 & 1.076970 & -1.625045 & .139095 \\ 9 & 8 & 0 & 1.141352 & -2.563470 & -.708216 \\ 10 & 8 & 0 & 1.829691 & -1.406881 & 1.121582 \\ 11 & 6 & 0 & 1.071922 & 1.628422 & -.138991 \\ 12 & 8 & 0 & 1.826153 & 1.411822 & -1.120652 \\ 13 & 8 & 0 & 1.132673 & 2.567741 & .707622 \\ 14 & 1 & 0 & -1.390799 & 2.468075 & -.034184 \\ 15 & 1 & 0 & -3.573338 & 1.253430 & -.029627 \\ 16 & 1 & 0 & -3.569373 & -1.264623 & .029253\end{array}$

$1,2-\mathrm{HO}_{2} \mathrm{CC}_{6} \mathrm{H}_{4} \mathrm{CO}_{2}^{-}$

$\mathrm{B} 3 \mathrm{LYP} /$ aug-cc-pvdz $=-608.951521$

$\mathrm{zpe}=0.116567$

thermal correction $=0.125177$

$\begin{array}{llrrrr}1 & 6 & 0 & .069012 & -1.437702 & .000000 \\ 2 & 6 & 0 & -1.158333 & -.779192 & .000000 \\ 3 & 6 & 0 & 1.276637 & -.718986 & .000000 \\ 4 & 6 & 0 & 1.221266 & .684367 & .000000 \\ 5 & 6 & 0 & .000000 & 1.356492 & .000000 \\ 6 & 6 & 0 & -1.206923 & .628852 & .000000 \\ 7 & 6 & 0 & -2.522469 & 1.291941 & .000000 \\ 8 & 6 & 0 & 2.642087 & -1.458759 & .000000 \\ 9 & 8 & 0 & 3.654463 & -.712511 & .000000 \\ 10 & 8 & 0 & 2.570128 & -2.714172 & .000000 \\ 11 & 8 & 0 & -3.613028 & .741444 & .000000 \\ 12 & 8 & 0 & -2.452902 & 2.664348 & .000000 \\ 13 & 1 & 0 & .134276 & -2.525411 & .000000 \\ 14 & 1 & 0 & -2.095349 & -1.335866 & .000000 \\ 15 & 1 & 0 & 2.169028 & 1.222123 & .000000 \\ 16 & 1 & 0 & -.028760 & 2.445338 & .000000 \\ 17 & 1 & 0 & -3.376140 & 2.958889 & .000000\end{array}$


$1,4-\mathrm{HO}_{2} \mathrm{CC}_{6} \mathrm{H}_{4} \mathrm{CO}_{2}^{-} / \mathrm{Cl}^{-}$

B3LYP/aug-cc-pvdz $=-1069.213797$ (radical at anion geom. -1069.153069 ) $\mathrm{zpe}=0.116534$

thermal correction $=0.127293$

$\begin{array}{rrrrrr}1 & 6 & 0 & 2.030033 & 1.205848 & -.000144 \\ 2 & 6 & 0 & .650943 & 1.426304 & -.000005 \\ 3 & 6 & 0 & 2.558767 & -.095705 & -.000022 \\ 4 & 6 & 0 & 1.655311 & -1.170597 & .000121 \\ 5 & 6 & 0 & .272822 & -.958698 & .000058 \\ 6 & 6 & 0 & -.249382 & .346751 & .000072 \\ 7 & 6 & 0 & -1.726775 & .628782 & .000235 \\ 8 & 6 & 0 & 4.090590 & -.335363 & -.000063 \\ 9 & 8 & 0 & 4.458819 & -1.541602 & .000781 \\ 10 & 8 & 0 & 4.808775 & .701974 & -.000974 \\ 11 & 8 & 0 & -2.178664 & 1.767532 & .000957 \\ 12 & 8 & 0 & -2.470288 & -.478499 & -.000417 \\ 13 & 1 & 0 & 2.736495 & 2.036094 & -.000423 \\ 14 & 1 & 0 & .243086 & 2.437405 & .000089 \\ 15 & 1 & 0 & 2.071383 & -2.178191 & .000360 \\ 16 & 1 & 0 & -.416702 & -1.802049 & -.000008 \\ 17 & 1 & 0 & -3.467433 & -.309247 & -.000190 \\ 18 & 17 & 0 & -5.518224 & -.591952 & -.000242\end{array}$

$1,4-\mathrm{HO}_{2} \mathrm{CC}_{6} \mathrm{H}_{4} \mathrm{CO}_{2}^{-} / \mathrm{Cl}^{-}$Transition structure

B3LYP/aug-cC-pvdz $=-1069.206086$

$\mathrm{zpe}=0.116927$

thermal correction $=0.127342$

$\begin{array}{lrrrrr}1 & 6 & 0 & -2.195643 & 1.498935 & .000000 \\ 2 & 6 & 0 & -.805223 & 1.607122 & .000000 \\ 3 & 6 & 0 & -2.826218 & .243382 & .000000 \\ 4 & 6 & 0 & -2.016971 & -.904151 & .000000 \\ 5 & 6 & 0 & -.623298 & -.808290 & .000000 \\ 6 & 6 & 0 & .000000 & .453258 & .000000 \\ 7 & 6 & 0 & 1.479138 & .616474 & .000000 \\ 8 & 6 & 0 & -4.373856 & .130708 & .000000 \\ 9 & 8 & 0 & -4.834234 & -1.041579 & .000000 \\ 10 & 8 & 0 & -4.998042 & 1.225402 & .000000 \\ 11 & 8 & 0 & 2.058624 & 1.690828 & .000000 \\ 12 & 8 & 0 & 2.151651 & -.562317 & .000000 \\ 13 & 1 & 0 & -2.834529 & 2.381861 & .000000 \\ 14 & 1 & 0 & -.317011 & 2.581673 & .000000 \\ 15 & 1 & 0 & -2.517278 & -1.872319 & .000000 \\ 16 & 1 & 0 & -.008454 & -1.707370 & .000000 \\ 17 & 1 & 0 & 3.107827 & -.375041 & .000000 \\ 18 & 17 & 0 & 6.806934 & -1.678359 & .000000\end{array}$




\begin{tabular}{|c|c|c|c|c|c|}
\hline \multicolumn{6}{|c|}{$\begin{array}{l}\text { В3LYP/aug-cC-pvdz }=-3183.160051 \\
\mathrm{zpe}=0.116665, \text { thermal correction }=0.127461\end{array}$} \\
\hline 1 & 6 & 0 & .604224 & 3.159539 & .000000 \\
\hline 2 & 6 & 0 & -.368099 & 2.157639 & .000000 \\
\hline 3 & 6 & 0 & 1.973269 & 2.844274 & .000000 \\
\hline 4 & 6 & 0 & 2.335263 & 1.487631 & .000000 \\
\hline 5 & 6 & 0 & 1.368546 & .477347 & .000000 \\
\hline 6 & 6 & 0 & .000000 & .800721 & .000000 \\
\hline 7 & 6 & 0 & -1.077989 & -.242778 & .000000 \\
\hline 8 & 6 & 0 & 3.049116 & 3.961071 & .000000 \\
\hline 9 & 8 & 0 & 4.247381 & 3.568992 & .000000 \\
\hline 10 & 8 & 0 & 2.610973 & 5.143881 & .000000 \\
\hline 11 & 8 & 0 & -2.270347 & .031108 & .000000 \\
\hline 12 & 8 & 0 & -.597339 & -1.493475 & .000000 \\
\hline 13 & 1 & 0 & .330906 & 4.214776 & .000000 \\
\hline 14 & 1 & 0 & -1.429962 & 2.404527 & .000000 \\
\hline 15 & 1 & 0 & 3.399177 & 1.250449 & .000000 \\
\hline 16 & 1 & 0 & 1.664102 & -.571088 & .000000 \\
\hline 17 & 1 & 0 & -1.315275 & -2.186706 & .000000 \\
\hline 18 & 35 & 0 & -2.339436 & -4.313962 & .000000 \\
\hline
\end{tabular}

$\left(1,4-\mathrm{HO}_{2} \mathrm{CC}_{6} \mathrm{H}_{4} \mathrm{CO}_{2}^{-}\right)_{2} \quad(8)$

B3LYP / 6-31+G $(\mathrm{d}, \mathrm{p})=-1217.767393$

zpe $=0.233592$, thermal correction $=0.251297$

\begin{tabular}{|c|c|c|c|c|c|}
\hline 1 & 6 & 0 & -.611601 & -1.540033 & 1.141652 \\
\hline 2 & 6 & 0 & -1.870914 & -.941641 & 1.098674 \\
\hline 3 & 6 & 0 & -.134294 & -2.301598 & .067955 \\
\hline 4 & 6 & 0 & -.989305 & -2.520610 & -1.023510 \\
\hline 5 & 6 & 0 & -2.258552 & -1.941885 & -1.064352 \\
\hline 6 & 6 & 0 & -2.698343 & -1.112328 & -.019534 \\
\hline 7 & 6 & 0 & -4.041236 & -.440956 & -.118100 \\
\hline 8 & 6 & 0 & 1.318371 & -2.791074 & .035107 \\
\hline 9 & 8 & 0 & 1.620938 & -3.673279 & -.793595 \\
\hline 10 & 8 & 0 & 2.100606 & -2.205870 & .868514 \\
\hline 11 & 8 & 0 & -5.003793 & -1.016177 & -.623463 \\
\hline 12 & 8 & 0 & -4.164302 & .786147 & .375422 \\
\hline 13 & 1 & 0 & .049220 & -1.378816 & 1.985719 \\
\hline 14 & 1 & 0 & -2.194922 & -.300725 & 1.911562 \\
\hline 15 & 1 & 0 & -.621075 & -3.125736 & -1.846610 \\
\hline 16 & 1 & 0 & -2.912687 & -2.095282 & -1.918078 \\
\hline 17 & 1 & 0 & -3.298690 & 1.327415 & .544524 \\
\hline 18 & 6 & 0 & 1.870791 & .941470 & 1.098677 \\
\hline 19 & 6 & 0 & .611502 & 1.539974 & 1.141612 \\
\hline 20 & 6 & 0 & 2.698285 & 1.112132 & -.019471 \\
\hline 21 & 6 & 0 & 2.258613 & 1.941798 & -1.064258 \\
\hline 22 & 6 & 0 & .989446 & 2.520682 & -1.023423 \\
\hline 23 & 6 & 0 & .134329 & 2.301683 & .067959 \\
\hline 24 & 6 & 0 & -1.318271 & 2.791407 & .035095 \\
\hline 25 & 6 & 0 & 4.041130 & .440662 & -.118039 \\
\hline 26 & 8 & 0 & 4.164147 & -.786451 & .375360 \\
\hline 27 & 8 & 0 & 5.003702 & 1.015873 & -.623436 \\
\hline 28 & 8 & 0 & -1.620523 & 3.673993 & -.793330 \\
\hline 29 & 8 & 0 & -2.100731 & 2.206054 & .868145 \\
\hline 30 & 1 & 0 & 2.194714 & .300569 & 1.911620 \\
\hline 31 & 1 & 0 & -.049343 & 1.378769 & 1.985669 \\
\hline 32 & 1 & 0 & 2.912810 & 2.095224 & -1.917929 \\
\hline 33 & 1 & 0 & .621381 & 3.125955 & -1.846493 \\
\hline 34 & 1 & 0 & 3.298527 & -1.327807 & .544823 \\
\hline
\end{tabular}


$\left(1,4-\mathrm{HO}_{2} \mathrm{CC}_{6} \mathrm{H}_{4} \mathrm{CO}_{2}^{-}\right)_{2}$ Transion Structure

$\mathrm{B} 3 \mathrm{LYP} / 6-31+\mathrm{G}(\mathrm{d}, \mathrm{p})=-1217.754730, \mathrm{zpe}=0.233982$, thermal correction $=0.251500$

\begin{tabular}{|c|c|c|c|c|c|}
\hline 1 & 6 & 0 & -1.441705 & -.517231 & .034931 \\
\hline 2 & 6 & 0 & -2.360346 & .525140 & -.071176 \\
\hline 3 & 6 & 0 & -1.866293 & -1.852384 & .051937 \\
\hline 4 & 6 & 0 & -3.238884 & -2.122913 & -.055416 \\
\hline 5 & 6 & 0 & -4.164158 & -1.086870 & -.156671 \\
\hline 6 & 6 & 0 & -3.738316 & .254895 & -.159375 \\
\hline 7 & 6 & 0 & -4.778112 & 1.318723 & -.252735 \\
\hline 8 & 6 & 0 & -.839199 & -2.994075 & .188412 \\
\hline 9 & 8 & 0 & -1.264869 & -4.160751 & .054015 \\
\hline 10 & 8 & 0 & .341707 & -2.598506 & .441243 \\
\hline 11 & 8 & 0 & -5.961719 & 1.105384 & -.448552 \\
\hline 12 & 8 & 0 & -4.375271 & 2.613115 & -.102555 \\
\hline 13 & 1 & 0 & -.381339 & -.308896 & .108150 \\
\hline 14 & 1 & 0 & -1.961386 & 1.538520 & -.091463 \\
\hline 15 & 1 & 0 & -3.552122 & -3.162474 & -.053766 \\
\hline 16 & 1 & 0 & -5.227908 & -1.289972 & -.231576 \\
\hline 17 & 1 & 0 & -3.426142 & 2.664918 & .087946 \\
\hline 18 & 6 & 0 & 2.248098 & -.172507 & .873725 \\
\hline 19 & 6 & 0 & 1.852805 & 1.164777 & .967784 \\
\hline 20 & 6 & 0 & 3.236620 & -.556577 & -.046632 \\
\hline 21 & 6 & 0 & 3.834551 & .437748 & -.841875 \\
\hline 22 & 6 & 0 & 3.425907 & 1.767967 & -.747312 \\
\hline 23 & 6 & 0 & 2.417843 & 2.153773 & .150857 \\
\hline 24 & 6 & 0 & 1.926831 & 3.621790 & .220992 \\
\hline 25 & 6 & 0 & 3.715634 & -1.973749 & -.189307 \\
\hline 26 & 8 & 0 & 2.897794 & -2.977066 & .136898 \\
\hline 27 & 8 & 0 & 4.852599 & -2.223591 & -.583897 \\
\hline 28 & 8 & 0 & 2.618300 & 4.462990 & -.415053 \\
\hline 29 & 8 & 0 & .880550 & 3.815283 & .900782 \\
\hline 30 & 1 & 0 & 1.769785 & -.920427 & 1.496630 \\
\hline 31 & 1 & 0 & 1.079483 & 1.470404 & 1.666134 \\
\hline 32 & 1 & 0 & 4.616184 & .141945 & -1.535932 \\
\hline 33 & 1 & 0 & 3.870888 & 2.541493 & -1.366295 \\
\hline 34 & 1 & 0 & 1.912179 & -2.781431 & .248299 \\
\hline
\end{tabular}

$2,6-\mathrm{C}_{10} \mathrm{H}_{6}\left(\mathrm{CO}_{2}^{-}\right)_{2}$

$\mathrm{B} 3 L Y P / 6-31+G(d, p)=-761.927728, z p e=0.149782$, thermal correction $=0.161370$

\begin{tabular}{|c|c|c|c|c|c|}
\hline 1 & 6 & 0 & -.252227 & -2.556376 & .000000 \\
\hline 2 & 6 & 0 & 1.141529 & -2.265400 & .000000 \\
\hline 3 & 6 & 0 & -1.141531 & -1.496280 & .000000 \\
\hline 4 & 6 & 0 & -.703659 & -.141335 & .000000 \\
\hline 5 & 6 & 0 & .703657 & .141334 & .000000 \\
\hline 6 & 6 & 0 & 1.603308 & -.964962 & .000000 \\
\hline 7 & 6 & 0 & -1.603310 & .964961 & .000000 \\
\hline 8 & 6 & 0 & -1.141531 & 2.265399 & .000000 \\
\hline 9 & 6 & 0 & .252225 & 2.556376 & .000000 \\
\hline 10 & 6 & 0 & 1.141529 & 1.496279 & .000000 \\
\hline 11 & 8 & 0 & 1.996013 & 4.194390 & .000000 \\
\hline 12 & 6 & 0 & -.745110 & -4.024896 & .000000 \\
\hline 13 & 1 & 0 & 1.821856 & -3.112023 & .000000 \\
\hline 14 & 8 & 0 & -1.996012 & -4.194395 & .000000 \\
\hline 15 & 8 & 0 & -.160207 & 4.907835 & .000000 \\
\hline 16 & 1 & 0 & -2.204458 & -1.725677 & .000000 \\
\hline 17 & 8 & 0 & .160214 & -4.907827 & .000000 \\
\hline 18 & 1 & 0 & 2.673606 & -.759563 & .000000 \\
\hline 19 & 1 & 0 & -2.673607 & .759562 & .000000 \\
\hline 20 & 1 & 0 & -1.821859 & 3.112021 & .000000 \\
\hline 21 & 6 & 0 & .745111 & 4.024896 & .000000 \\
\hline 22 & 1 & 0 & 2.204455 & 1 & \\
\hline
\end{tabular}




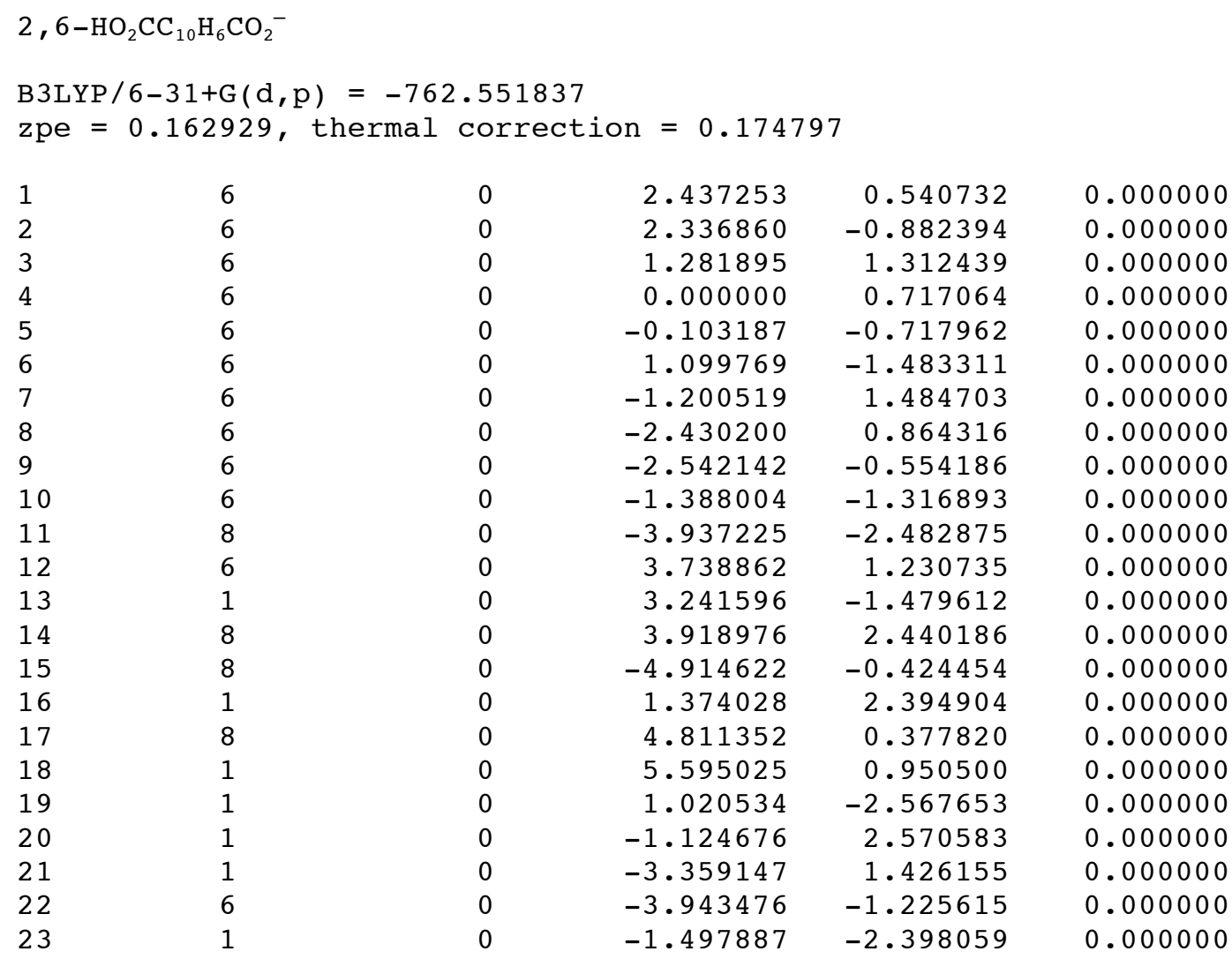

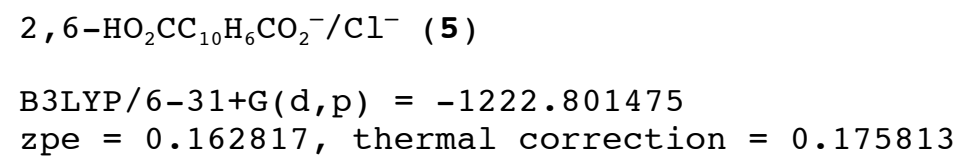

$\begin{array}{lrrrrr}1 & 6 & 0 & -1.032271 & -1.054849 & .000000 \\ 2 & 6 & 0 & .263411 & -1.646097 & .000000 \\ 3 & 6 & 0 & -1.143988 & .325126 & .000000 \\ 4 & 6 & 0 & .000000 & 1.165169 & .000000 \\ 5 & 6 & 0 & 1.305609 & .567818 & .000000 \\ 6 & 6 & 0 & 1.391842 & -.854858 & .000000 \\ 7 & 6 & 0 & -.088811 & 2.587564 & .000000 \\ 8 & 6 & 0 & 1.046605 & 3.369353 & .000000 \\ 9 & 6 & 0 & 2.345850 & 2.787895 & .000000 \\ 10 & 6 & 0 & 2.452366 & 1.409020 & .000000 \\ 11 & 8 & 0 & 4.714036 & 3.093844 & .000000 \\ 12 & 6 & 0 & -2.287441 & -1.888134 & .000000 \\ 13 & 1 & 0 & .339392 & -2.727715 & .000000 \\ 14 & 8 & 0 & -3.405930 & -1.386723 & .000000 \\ 15 & 8 & 0 & 3.379453 & 4.935001 & .000000 \\ 16 & 1 & 0 & -2.138266 & .763644 & .000000 \\ 17 & 8 & 0 & -2.033485 & -3.192617 & .000000 \\ 18 & 1 & 0 & -2.854846 & -3.795681 & .000000 \\ 19 & 1 & 0 & 2.379209 & -1.312371 & .000000 \\ 20 & 17 & 0 & -4.087089 & -5.398092 & .000000 \\ 21 & 1 & 0 & -1.076992 & 3.045448 & .000000 \\ 22 & 1 & 0 & .994787 & 4.453886 & .000000 \\ 23 & 6 & 0 & 3.605938 & 3.693138 & .000000 \\ 24 & 1 & 0 & 3.449980 & .977444 & .000000\end{array}$


2, 6- $\mathrm{HO}_{2} \mathrm{CC}_{10} \mathrm{H}_{6} \mathrm{CO}_{2}^{-} / \mathrm{Cl}^{-}$Transition structure

B3LYP / 6-31+G $(\mathrm{d}, \mathrm{p})=-1222.789951$

$\mathrm{zpe}=0.163339$, thermal correction $=0.176049$

\begin{tabular}{|c|c|c|c|c|c|}
\hline 1 & 6 & 0 & .000000 & 1.187991 & .000000 \\
\hline 2 & 6 & 0 & 1.293566 & .589893 & .000000 \\
\hline 3 & 6 & 0 & -1.127014 & .379959 & .000000 \\
\hline 4 & 6 & 0 & -1.023529 & -1.032505 & .000000 \\
\hline 5 & 6 & 0 & .279984 & -1.637080 & .000000 \\
\hline 6 & 6 & 0 & 1.419953 & -.781200 & .000000 \\
\hline 7 & 6 & 0 & -2.165226 & -1.885240 & .000000 \\
\hline 8 & 6 & 0 & -2.021940 & -3.255542 & .000000 \\
\hline 9 & 6 & 0 & -.735744 & -3.864911 & .000000 \\
\hline 10 & 6 & 0 & .384448 & -3.053680 & .000000 \\
\hline 11 & 8 & 0 & .568271 & -5.861654 & .000000 \\
\hline 12 & 6 & 0 & -.188344 & 2.663130 & .000000 \\
\hline 13 & 1 & 0 & 2.170054 & 1.227889 & .000000 \\
\hline 14 & 8 & 0 & -1.271112 & 3.228101 & .000000 \\
\hline 15 & 8 & 0 & -1.700649 & -6.040665 & .000000 \\
\hline 16 & 1 & 0 & -2.106443 & .850118 & .000000 \\
\hline 17 & 8 & 0 & .981376 & 3.347548 & .000000 \\
\hline 18 & 1 & 0 & .785330 & 4.302071 & .000000 \\
\hline 19 & 1 & 0 & 2.407557 & -1.236629 & .000000 \\
\hline 20 & 17 & 0 & 2.337772 & 8.409789 & .000000 \\
\hline 21 & 1 & 0 & -3.154191 & -1.429844 & .000000 \\
\hline 22 & 1 & 0 & -2.878723 & -3.922328 & .000000 \\
\hline 23 & 6 & 0 & -.607392 & -5.412293 & .000000 \\
\hline 24 & 1 & 0 & 1.358629 & -3.535442 & .000000 \\
\hline
\end{tabular}

$2,6-\mathrm{C}_{14} \mathrm{H}_{8}\left(\mathrm{CO}_{2}^{-}\right)_{2}$

B3LYP $/ 6-31+G(d, p)=-915.586754$

$\mathrm{zpe}=0.196049$, thermal correction $=0.209933$

\begin{tabular}{|c|c|c|c|c|c|}
\hline 1 & 6 & 0 & 0.460745 & -5.240083 & -0.000000 \\
\hline 2 & 6 & 0 & -0.011531 & -3.764632 & -0.000000 \\
\hline 3 & 1 & 0 & 1.946579 & -2.963077 & -0.000000 \\
\hline 4 & 6 & 0 & 0.886487 & -2.722022 & -0.000000 \\
\hline 5 & 6 & 0 & -1.864034 & -2.164297 & -0.000000 \\
\hline 6 & 6 & 0 & 0.465001 & -1.352985 & -0.000000 \\
\hline 7 & 6 & 0 & -1.411469 & -3.460323 & -0.000000 \\
\hline 8 & 6 & 0 & -0.951835 & -1.058590 & -0.000000 \\
\hline 9 & 6 & 0 & 1.372740 & -0.280263 & -0.000000 \\
\hline 10 & 1 & 0 & -2.094070 & -4.304817 & -0.000000 \\
\hline 11 & 1 & 0 & -2.439951 & 0.498761 & 0.000000 \\
\hline 12 & 1 & 0 & -2.932565 & -1.950853 & 0.000000 \\
\hline 13 & 6 & 0 & 0.951835 & 1.058591 & 0.000000 \\
\hline 14 & 1 & 0 & 2.439952 & -0.498761 & -0.000000 \\
\hline 15 & 6 & 0 & 1.864034 & 2.164297 & 0.000000 \\
\hline 16 & 6 & 0 & -0.465000 & 1.352985 & 0.000000 \\
\hline 17 & 1 & 0 & -1.946579 & 2.963078 & 0.000000 \\
\hline 18 & 6 & 0 & -1.372739 & 0.280263 & 0.000000 \\
\hline 19 & 6 & 0 & 1.411469 & 3.460324 & 0.000000 \\
\hline 20 & 1 & 0 & 2.932565 & 1.950854 & -0.000000 \\
\hline 21 & 1 & 0 & 2.094072 & 4.304817 & 0.000000 \\
\hline 22 & 6 & 0 & 0.011531 & 3.764632 & 0.000000 \\
\hline 23 & 6 & 0 & -0.460746 & 5.240083 & 0.000000 \\
\hline 24 & 6 & 0 & -0.886487 & 2.722022 & 0.000000 \\
\hline 25 & 8 & 0 & -1.707946 & 5.423943 & 0.000000 \\
\hline 26 & 8 & 0 & 0.460745 & 6.104767 & 0.000000 \\
\hline 27 & 8 & 0 & 1.707946 & -5.423944 & -0.000000 \\
\hline 28 & 8 & 0 & -0.460746 & -6.104767 & -0.000000 \\
\hline
\end{tabular}




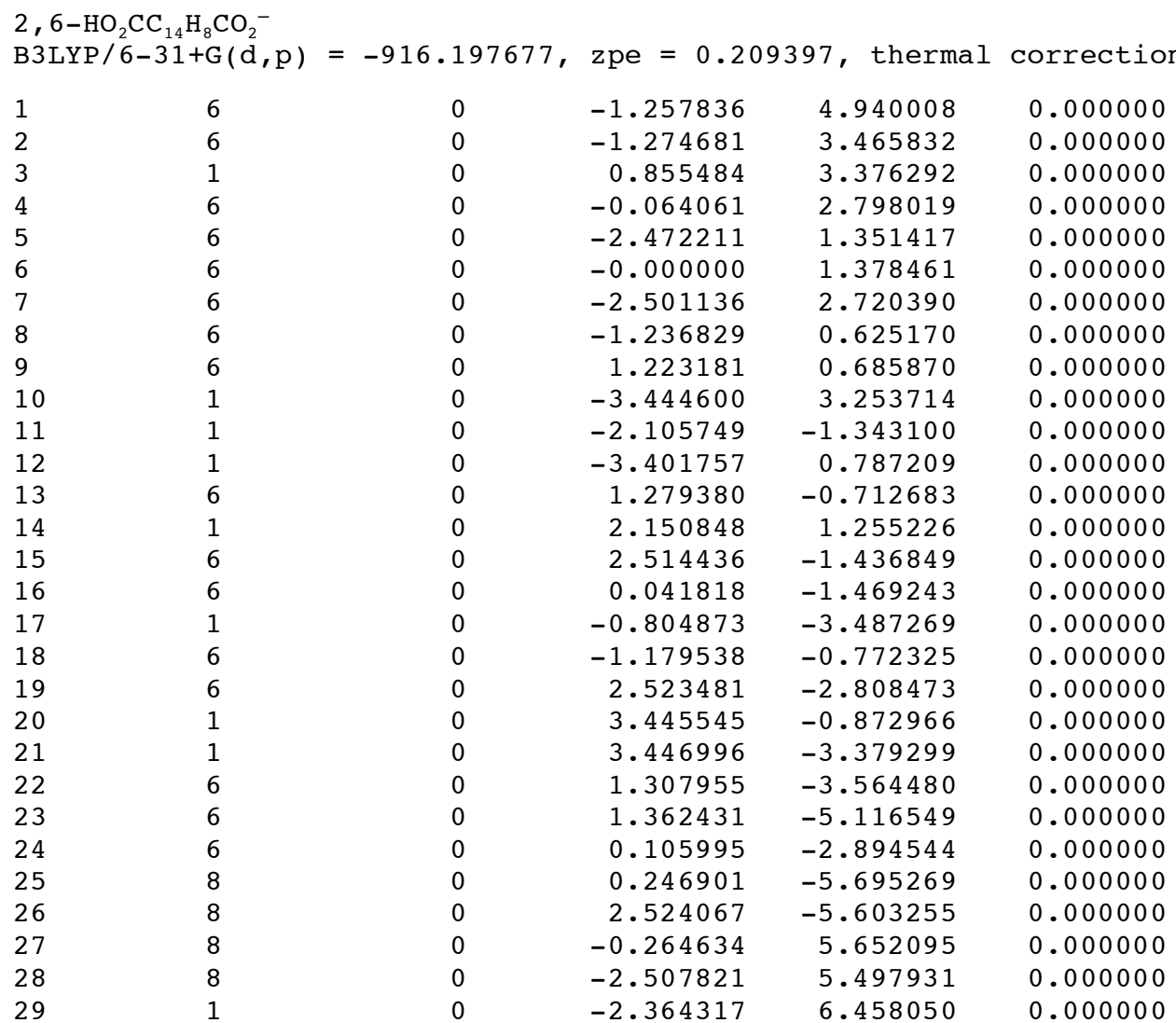

$2,6-\mathrm{HO}_{2} \mathrm{CC}_{14} \mathrm{H}_{8} \mathrm{CO}_{2}^{-} / \mathrm{Cl}^{-}$(6)

$\mathrm{B} 3 \mathrm{LYP} / 6-31+\mathrm{G}(\mathrm{d}, \mathrm{p})=-1376.455302, \mathrm{zpe}=0.209023$, thermal correction $=0.224292$

$\begin{array}{rrrrrr}1 & 6 & 0 & -4.133936 & 0.494002 & -0.000105 \\ 2 & 6 & 0 & -2.713167 & -0.013340 & 0.000062 \\ 3 & 1 & 0 & -1.921526 & 1.955872 & 0.000298 \\ 4 & 6 & 0 & -1.679863 & 0.896592 & 0.000180 \\ 5 & 6 & 0 & -1.115571 & -1.852439 & 0.000138 \\ 6 & 6 & 0 & -0.313301 & 0.481656 & 0.000139 \\ 7 & 6 & 0 & -2.415272 & -1.415115 & 0.000086 \\ 8 & 6 & 0 & -0.016006 & -0.933735 & 0.000122 \\ 9 & 6 & 0 & 0.751303 & 1.398167 & 0.000168 \\ 10 & 1 & 0 & -3.242003 & -2.116314 & 0.000145 \\ 11 & 1 & 0 & 1.547970 & -2.413816 & 0.000109 \\ 12 & 1 & 0 & -0.897346 & -2.918542 & 0.000173 \\ 13 & 6 & 0 & 2.090272 & 0.982810 & 0.000128 \\ 14 & 1 & 0 & 0.526725 & 2.463585 & 0.000217 \\ 15 & 6 & 0 & 3.189219 & 1.902104 & 0.000136 \\ 16 & 6 & 0 & 2.390002 & -0.433998 & 0.000076 \\ 17 & 1 & 0 & 4.008278 & -1.907590 & -0.000068 \\ 18 & 6 & 0 & 1.323354 & -1.348627 & 0.000115 \\ 19 & 6 & 0 & 4.486124 & 1.454147 & 0.000059 \\ 20 & 1 & 0 & 2.969828 & 2.968898 & 0.000179 \\ 21 & 1 & 5.329457 & 2.137944 & 0.000047 \\ 22 & 6 & 0 & 4.795152 & 0.055682 & -0.000029 \\ 23 & 6 & 0 & -274834 & -0.409782 & -0.000170 \\ 24 & 6 & 0 & -0.849567 & -0.000004 \\ 25 & 8 & 0 & 3.759450 & -0.840 \\ 26 & 8 & 0 & 6.459064 & -1.655208 & -0.000079 \\ 27 & 8 & 0 & 7.129507 & 0.518933 & -0.000337 \\ 28 & 8 & 0 & -4.396438 & 1.690781 & -0.000218 \\ 29 & 1 & 0 & -5.020813 & -0.491700 & -0.000159 \\ 30 & 17 & 0 & -6.004425 & -0.203723 & 0.000107 \\ & 6 & -7.983710 & -0.171771 & -0.000087\end{array}$


2, 6- $\mathrm{HO}_{2} \mathrm{CC}_{14} \mathrm{H}_{8} \mathrm{CO}_{2}^{-} / \mathrm{Cl}^{-}$Transition structure

B3LYP / 6-31+G $(\mathrm{d}, \mathrm{p})=-1376.440529$

zpe $=0.209702$, thermal correction $=0.224687$

\begin{tabular}{|c|c|c|c|c|c|}
\hline 1 & 6 & 0 & 3.788601 & 0.711885 & 0.000000 \\
\hline 2 & 6 & 0 & 2.411623 & 0.150072 & 0.000000 \\
\hline 3 & 1 & 0 & 1.547821 & 2.095052 & 0.000000 \\
\hline 4 & 6 & 0 & 1.346986 & 1.027517 & 0.000000 \\
\hline 5 & 6 & 0 & 0.885373 & -1.742221 & 0.000000 \\
\hline 6 & 6 & 0 & -0.000000 & 0.564081 & 0.000000 \\
\hline 7 & 6 & 0 & 2.169124 & -1.263222 & 0.000000 \\
\hline 8 & 6 & 0 & -0.245254 & -0.861827 & 0.000000 \\
\hline 9 & 6 & 0 & -1.094793 & 1.444711 & 0.000000 \\
\hline 10 & 1 & 0 & 3.014694 & -1.941181 & 0.000000 \\
\hline 11 & 1 & 0 & -1.755693 & -2.395367 & 0.000000 \\
\hline 12 & 1 & 0 & 0.704614 & -2.814646 & 0.000000 \\
\hline 13 & 6 & 0 & -2.416903 & 0.981944 & 0.000000 \\
\hline 14 & 1 & 0 & -0.907381 & 2.517031 & 0.000000 \\
\hline 15 & 6 & 0 & -3.546022 & 1.863046 & 0.000000 \\
\hline 16 & 6 & 0 & -2.665485 & -0.445747 & 0.000000 \\
\hline 17 & 1 & 0 & -4.230704 & -1.975567 & 0.000000 \\
\hline 18 & 6 & 0 & -1.567692 & -1.323540 & 0.000000 \\
\hline 19 & 6 & 0 & -4.825400 & 1.368260 & 0.000000 \\
\hline 20 & 1 & 0 & -3.364214 & 2.936552 & 0.000000 \\
\hline 21 & 1 & 0 & -5.694011 & 2.019550 & 0.000000 \\
\hline 22 & 6 & 0 & -5.083358 & -0.040165 & 0.000000 \\
\hline 23 & 6 & 0 & -6.546858 & -0.557307 & 0.000000 \\
\hline 24 & 6 & 0 & -4.017470 & -0.909913 & 0.000000 \\
\hline 25 & 8 & 0 & -6.683187 & -1.807691 & 0.000000 \\
\hline 26 & 8 & 0 & -7.429433 & 0.343407 & 0.000000 \\
\hline 27 & 8 & 0 & 4.056950 & 1.902504 & 0.000000 \\
\hline 28 & 8 & 0 & 4.747670 & -0.245296 & 0.000000 \\
\hline 29 & 1 & 0 & 5.619178 & 0.189389 & 0.000000 \\
\hline 30 & 17 & 0 & 10.351463 & -0.469391 & 0.000000 \\
\hline
\end{tabular}

\section{$1,3-\mathrm{HO}_{2} \mathrm{CC}_{5} \mathrm{H}_{6} \mathrm{CO}_{2}^{-}$}

B3LYP $/ 6-31+G(d, p)=-571.898089, z p e=0.132566$

$\begin{array}{rrrrrr}1 & 6 & 0 & -0.031641 & -0.677547 & -1.027856 \\ 2 & 6 & 0 & 0.887671 & 0.065881 & -0.005197 \\ 3 & 6 & 0 & -0.031645 & -0.510414 & 1.120110 \\ 4 & 6 & 0 & 2.375425 & 0.117060 & -0.009229 \\ 5 & 1 & 0 & 0.012152 & -1.771441 & -1.019632 \\ 6 & 1 & 0 & -0.051528 & -0.275623 & -2.044995 \\ 7 & 6 & 0 & -1.001824 & -0.009949 & 0.000652 \\ 8 & 6 & 0 & -0.086244 & 1.262880 & -0.098344 \\ 9 & 6 & 0 & -2.540146 & -0.029620 & 0.002352 \\ 10 & 1 & 0 & -0.110871 & 1.943476 & 0.758531 \\ 11 & 1 & 0 & -0.110716 & 1.802748 & -1.050191 \\ 12 & 1 & 0 & -0.051558 & 0.044040 & 2.062811 \\ 13 & 1 & 0 & 0.012114 & -1.592421 & 1.281194 \\ 14 & 8 & 0 & 3.073765 & 1.113946 & -0.086602 \\ 15 & 8 & 0 & 2.946494 & -1.123975 & 0.087572 \\ 16 & 1 & 0 & 3.906301 & -0.974173 & 0.075983 \\ 17 & 8 & 0 & -3.074675 & 0.064653 & 1.139538 \\ 18 & 8 & 0 & -3.075017 & -0.115418 & -1.135338\end{array}$




\begin{tabular}{|c|c|c|c|c|c|}
\hline \multicolumn{3}{|c|}{$\left(1,3-\mathrm{HO}_{2} \mathrm{CC}_{5} \mathrm{H}_{6} \mathrm{CO}_{2}^{-}\right)_{4} \quad(10)$} & $16-31+C 1$ & \multicolumn{2}{|c|}{$=-2287.387303$} \\
\hline 1 & 6 & 0 & 6.298755 & -2.902150 & 0.412956 \\
\hline 2 & 6 & 0 & 4.772537 & -2.662942 & 0.250300 \\
\hline 3 & 6 & 0 & 3.667532 & -3.695266 & 0.345122 \\
\hline 4 & 6 & 0 & 4.945422 & -1.429552 & 1.200966 \\
\hline 5 & 1 & 0 & 6.796459 & -3.456095 & -0.392182 \\
\hline 6 & 1 & 0 & 6.641899 & -3.237604 & 1.397653 \\
\hline 7 & 6 & 0 & 6.157006 & -1.364983 & 0.210961 \\
\hline 8 & 6 & 0 & 5.113863 & -1.686725 & -0.914681 \\
\hline 9 & 6 & 0 & 7.284166 & -0.331016 & 0.170795 \\
\hline 10 & 1 & 0 & 5.515242 & -2.145692 & -1.827217 \\
\hline 11 & 1 & 0 & 4.376213 & -0.913024 & -1.138790 \\
\hline 12 & 1 & 0 & 4.197928 & -0.637233 & 1.117577 \\
\hline 13 & 1 & 0 & 5.186999 & -1.661879 & 2.245619 \\
\hline 14 & 8 & 0 & 3.814423 & -4.726238 & 1.006407 \\
\hline 15 & 8 & 0 & 2.586960 & -3.341722 & -0.312436 \\
\hline 16 & 1 & 0 & 1.700973 & -3.915752 & -0.181358 \\
\hline 17 & 8 & 0 & 8.462756 & -0.760540 & 0.206255 \\
\hline 18 & 8 & 0 & 6.942108 & 0.901014 & 0.099855 \\
\hline 19 & 1 & 0 & 5.613804 & 1.568189 & 0.086152 \\
\hline 20 & 8 & 0 & 0.318596 & -4.331805 & 0.034744 \\
\hline 21 & 8 & 0 & 4.656337 & 1.987289 & 0.203805 \\
\hline 22 & 6 & 0 & -1.994805 & -4.508844 & -0.497793 \\
\hline 23 & 6 & 0 & -0.520381 & -4.709404 & -0.855409 \\
\hline 24 & 8 & 0 & -0.271900 & -5.226332 & -1.968787 \\
\hline 25 & 6 & 0 & 3.014491 & 3.639821 & -0.191028 \\
\hline 26 & 6 & 0 & 4.416766 & 3.125108 & -0.424280 \\
\hline 27 & 8 & 0 & 5.221958 & 3.737153 & -1.125805 \\
\hline 28 & 6 & 0 & -2.636736 & -3.942278 & 0.806458 \\
\hline 29 & 6 & 0 & -3.088648 & -5.623526 & -0.462431 \\
\hline 30 & 6 & 0 & -3.037694 & -3.637657 & -1.278437 \\
\hline 31 & 6 & 0 & 1.654927 & 2.919784 & -0.479033 \\
\hline 32 & 6 & 0 & 2.388501 & 4.915582 & -0.810601 \\
\hline 33 & 6 & 0 & 2.384693 & 4.089173 & 1.168163 \\
\hline 34 & 1 & 0 & 1.423415 & 2.046219 & 0.135660 \\
\hline 35 & 1 & 0 & 1.412026 & 2.745860 & -1.531965 \\
\hline 36 & 1 & 0 & 2.200371 & 4.892592 & -1.890193 \\
\hline 37 & 1 & 0 & 2.808364 & 5.876173 & -0.492546 \\
\hline 38 & 6 & 0 & 1.265711 & 4.313226 & 0.101687 \\
\hline 39 & 1 & 0 & 2.206556 & 3.299413 & 1.905062 \\
\hline 40 & 1 & 0 & 2.806225 & 4.991076 & 1.626246 \\
\hline 41 & 1 & 0 & -2.486641 & -2.879636 & 1.009486 \\
\hline 42 & 1 & 0 & -2.519051 & -4.552197 & 1.709820 \\
\hline 43 & 1 & 0 & -3.006076 & -6.363498 & 0.343474 \\
\hline 44 & 1 & 0 & -3.332188 & -6.096759 & -1.419955 \\
\hline 45 & 6 & 0 & -3.844615 & -4.314249 & -0.120993 \\
\hline 46 & 1 & 0 & -3.277810 & -3.974087 & -2.293816 \\
\hline 47 & 1 & 0 & -2.921570 & -2.552695 & -1.217484 \\
\hline 48 & 6 & 0 & -5.326125 & -4.220140 & 0.180415 \\
\hline 49 & 6 & 0 & -0.113552 & 4.930332 & 0.323911 \\
\hline 50 & 8 & 0 & -6.007433 & -5.253089 & 0.239321 \\
\hline 51 & 8 & 0 & -5.865136 & -3.038324 & 0.372745 \\
\hline 52 & 1 & 0 & -5.284002 & -2.125395 & 0.316126 \\
\hline 53 & 8 & 0 & -1.091131 & 4.299772 & -0.221343 \\
\hline 54 & 8 & 0 & -0.169508 & 5.990159 & 0.984046 \\
\hline 55 & 8 & 0 & -4.753429 & -0.837827 & 0.104317 \\
\hline 56 & 6 & 0 & -4.690819 & 1.524223 & 0.429893 \\
\hline 57 & 6 & 0 & -4.953821 & 0.112309 & 0.949844 \\
\hline 58 & 8 & 0 & -5.385861 & 0.002287 & 2.115544 \\
\hline 59 & 6 & 0 & -4.118092 & 2.743732 & 1.220712 \\
\hline 60 & 6 & 0 & -5.821885 & 2.487896 & -0.062234 \\
\hline 61 & 6 & 0 & -3.849757 & 2.024801 & -0.787440 \\
\hline 62 & 1 & 0 & -4.204465 & 1.719883 & -1.777913 \\
\hline 63 & 1 & 0 & -2.762724 & 1.956989 & -0.701460 \\
\hline 64 & 1 & 0 & -3.049261 & 2.724712 & 1.449535 \\
\hline 65 & 1 & 0 & -4.716976 & 3.092757 & 2.069143 \\
\hline 66 & 6 & 0 & -4.515847 & 3.310673 & -0.183739 \\
\hline 67 & 1 & 0 & -6.541178 & 2.817019 & 0.696306 \\
\hline 68 & 1 & 0 & -6.318330 & 2.215413 & -1.000743 \\
\hline 69 & 6 & 0 & -4.448064 & 4.741911 & -0.675835 \\
\hline 70 & 8 & 0 & -5.481785 & 5.309967 & -1.050242 \\
\hline 71 & 8 & 0 & -3.289777 & 5.363402 & -0.690230 \\
\hline 72 & 1 & 0 & -2.384816 & 4.864881 & -0.397300 \\
\hline
\end{tabular}




\begin{tabular}{|c|c|c|c|c|c|}
\hline \multicolumn{2}{|c|}{$\mathrm{HF} / 6-31+\mathrm{G}(\mathrm{d}, \mathrm{p})$} & \multirow[t]{2}{*}{ Geometry } & & & \\
\hline 1 & 6 & & -6.383725 & -2.895204 & -0.434978 \\
\hline 2 & 6 & 0 & -4.878653 & -2.704298 & -0.179241 \\
\hline 3 & 6 & 0 & -3.815205 & -3.770420 & -0.185601 \\
\hline 4 & 6 & 0 & -4.947205 & -1.470584 & -1.116213 \\
\hline 5 & 1 & 0 & -6.943804 & -3.434119 & 0.323079 \\
\hline 6 & 1 & 0 & -6.673567 & -3.213320 & -1.431329 \\
\hline 7 & 6 & 0 & -6.206815 & -1.377058 & -0.216119 \\
\hline 8 & 6 & 0 & -5.260865 & -1.735129 & 0.962799 \\
\hline 9 & 6 & 0 & -7.287391 & -0.297368 & -0.233767 \\
\hline 10 & 1 & 0 & -5.727656 & -2.182017 & 1.837103 \\
\hline 11 & 1 & 0 & -4.522838 & -0.994032 & 1.240706 \\
\hline 12 & 1 & 0 & -4.188692 & -0.711410 & -0.980200 \\
\hline 13 & 1 & 0 & -5.120663 & -1.679253 & -2.168672 \\
\hline 14 & 8 & 0 & -4.016800 & -4.873882 & -0.620296 \\
\hline 15 & 8 & 0 & -2.687711 & -3.349705 & 0.307882 \\
\hline 16 & 1 & 0 & -1.929532 & -3.970474 & 0.269385 \\
\hline 17 & 8 & 0 & -8.457401 & -0.685237 & -0.291284 \\
\hline 18 & 8 & 0 & -6.884373 & 0.881807 & -0.182132 \\
\hline 19 & 1 & 0 & -5.425798 & 1.826332 & -0.148430 \\
\hline 20 & 8 & 0 & -0.339523 & -4.610458 & 0.175674 \\
\hline 21 & 8 & 0 & -4.543221 & 2.221875 & -0.284350 \\
\hline 22 & 6 & 0 & 1.992426 & -4.634409 & 0.533650 \\
\hline 23 & 6 & 0 & 0.556999 & -4.873899 & 1.000243 \\
\hline 24 & 8 & 0 & 0.433825 & -5.335545 & 2.137094 \\
\hline 25 & 6 & 0 & -2.924053 & 3.861462 & 0.125800 \\
\hline 26 & 6 & 0 & -4.313885 & 3.337059 & 0.356975 \\
\hline 27 & 8 & 0 & -5.112196 & 3.901212 & 1.055501 \\
\hline 28 & 6 & 0 & 2.513375 & -3.941813 & -0.748633 \\
\hline 29 & 6 & 0 & 3.033486 & -5.749645 & 0.264431 \\
\hline 30 & 6 & 0 & 3.119893 & -3.887239 & 1.293031 \\
\hline 31 & 6 & 0 & -1.578822 & 3.156592 & 0.438656 \\
\hline 32 & 6 & 0 & -2.315191 & 5.140465 & 0.722969 \\
\hline 33 & 6 & 0 & -2.290407 & 4.281861 & -1.223330 \\
\hline 34 & 1 & 0 & -1.339375 & 2.280228 & -0.150154 \\
\hline 35 & 1 & 0 & -1.347911 & 3.000668 & 1.486598 \\
\hline 36 & 1 & 0 & -2.142647 & 5.141868 & 1.795027 \\
\hline 37 & 1 & 0 & -2.731030 & 6.085004 & 0.388427 \\
\hline 38 & 6 & 0 & -1.193164 & 4.528665 & -0.157258 \\
\hline 39 & 1 & 0 & -2.104582 & 3.488199 & -1.938465 \\
\hline 40 & 1 & 0 & -2.706346 & 5.162895 & -1.701553 \\
\hline 41 & 1 & 0 & 2.378629 & -2.870869 & -0.820095 \\
\hline 42 & 1 & 0 & 2.289603 & -4.436666 & -1.688352 \\
\hline 43 & 1 & 0 & 2.851192 & -6.385984 & -0.597398 \\
\hline 44 & 1 & 0 & 3.352995 & -6.330823 & 1.123147 \\
\hline 45 & 6 & 0 & 3.781464 & -4.434858 & 0.000677 \\
\hline 46 & 1 & 0 & 3.446005 & -4.334859 & 2.226555 \\
\hline 47 & 1 & 0 & 3.030766 & -2.810677 & 1.368891 \\
\hline 48 & 6 & 0 & 5.228557 & -4.342546 & -0.409035 \\
\hline 49 & 6 & 0 & 0.192116 & 5.127759 & -0.378863 \\
\hline 50 & 8 & 0 & 5.896274 & -5.341304 & -0.530608 \\
\hline 51 & 8 & 0 & 5.756010 & -3.171290 & -0.617120 \\
\hline 52 & 1 & 0 & 5.204822 & -2.357903 & -0.524727 \\
\hline 53 & 8 & 0 & 1.148441 & 4.469298 & 0.081848 \\
\hline 54 & 8 & 0 & 0.230229 & 6.204721 & -0.974139 \\
\hline 55 & 8 & 0 & 4.625700 & -0.832789 & -0.277019 \\
\hline 56 & 6 & 0 & 4.660611 & 1.518561 & -0.441299 \\
\hline 57 & 6 & 0 & 4.834319 & 0.130517 & -1.049536 \\
\hline 58 & 8 & 0 & 5.211184 & 0.087864 & -2.218728 \\
\hline 59 & 6 & 0 & 4.308421 & 2.839125 & -1.166957 \\
\hline 60 & 6 & 0 & 5.799623 & 2.303779 & 0.259918 \\
\hline 61 & 6 & 0 & 3.736421 & 1.995285 & 0.707057 \\
\hline 62 & 1 & 0 & 3.934105 & 1.575936 & 1.687564 \\
\hline 63 & 1 & 0 & 2.676063 & 2.047010 & 0.498170 \\
\hline 64 & 1 & 0 & 3.289361 & 2.950864 & -1.515316 \\
\hline 65 & 1 & 0 & 5.026590 & 3.191000 & -1.900524 \\
\hline 66 & 6 & 0 & 4.581645 & 3.237031 & 0.307830 \\
\hline 67 & 1 & 0 & 6.628933 & 2.616235 & -0.367484 \\
\hline 68 & 1 & 0 & 6.148119 & 1.907418 & 1.208340 \\
\hline 69 & 6 & 0 & 4.588106 & 4.618621 & 0.908651 \\
\hline 70 & 8 & 0 & 5.591420 & 5.070953 & 1.402743 \\
\hline 71 & 8 & 0 & 3.495169 & 5.328111 & 0.877222 \\
\hline 72 & 1 & 0 & 2.678552 & 4.939838 & 0.490250 \\
\hline
\end{tabular}




\begin{tabular}{|c|c|c|c|c|c|}
\hline 1 & 8 & 0 & 1.174192 & .848170 & .000000 \\
\hline 2 & 6 & 0 & .000000 & .553697 & .000000 \\
\hline 3 & 8 & 0 & -.991526 & 1.482861 & .000000 \\
\hline 4 & 1 & 0 & -.566383 & 2.356154 & .000000 \\
\hline 5 & 6 & 0 & -.564323 & -.861749 & .000000 \\
\hline 6 & 1 & 0 & -1.222284 & -.955353 & .876808 \\
\hline 7 & 1 & 0 & -1.222284 & -.955353 & -.876808 \\
\hline 8 & 7 & 0 & .413002 & -1.933004 & .000000 \\
\hline 9 & 1 & 0 & 1.022274 & -1.857177 & -.811576 \\
\hline 10 & 1 & 0 & 1.022274 & -1.857177 & .811576 \\
\hline
\end{tabular}

Deprotonated Glycine B3LYP/aug-cC-pvdz $=-283.928471$ $\mathrm{zpe}=0.066055$, thermal correction $=0.071978$

$\begin{array}{rrrrrr}1 & 8 & 0 & -0.537791 & 1.322023 & 0.036341 \\ 2 & 6 & 0 & -0.670569 & 0.065510 & 0.001037 \\ 3 & 8 & 0 & -1.726465 & -0.613615 & -0.086111 \\ 4 & 6 & 0 & 0.650668 & -0.760356 & 0.117740 \\ 5 & 1 & 0 & 0.632309 & -1.255073 & 1.103887 \\ 6 & 1 & 0 & 0.634503 & -1.564662 & -0.632230 \\ 7 & 7 & 0 & 1.893551 & 0.032695 & -0.004227 \\ 8 & 1 & 0 & 2.119103 & 0.109094 & -0.996032 \\ 9 & 1 & 0 & 1.592681 & 0.983584 & 0.239467\end{array}$

$\mathrm{HCl}$

B3LYP/aug-cC-pvdz $=-460.827703$

$\mathrm{zpe}=0.006656$, thermal correction $=0.009960$

$\begin{array}{rrrrrr}1 & 1 & 0 & 0.000000 & 0.000000 & -1.223065 \\ 2 & 17 & 0 & 0.000000 & 0.000000 & 0.071945\end{array}$

$\mathrm{Cl}^{-} \quad \mathrm{B} 3 \mathrm{LYP} / \mathrm{aug}-\mathrm{CC}-\mathrm{pvdz}=-460.298181$

$\mathrm{Br}^{-} \quad \mathrm{B} 3 \mathrm{LYP} / \mathrm{aug}-\mathrm{CC}-\mathrm{pvdz}=-2574.248077$

Complete Reference $7 a$

Frisch, M. J.; Trucks, G. W.; Schlegel, H. B.; Scuseria, G. E.; Robb, M. A.; Cheeseman, J. R.; Montgomery, J. A.; Vreven, T.; Kudin, K. N.; Burant, J. C.; Millam, J. M.; Iyengar, S. S.; Tomasi, J.; Barone, V.; Mennucci, B.; Cossi, M.; Scalmani, G.; Rega, N.; Petersson, G. A.; Nakatsuji, H.; Hada, M.; Ehara, M.; Toyota, K.; Fukuda, R.; Hasegawa, J.; Ishida, M.; Nakajima, T.; Honda, Y.; Kitao, O.; Nakai, H.; Klene, M.; X., L.; Knox, J. E.; Hratchian, H. P.; Cross, J. B.; Adamo, C.; Jaramillo, J.; Gomperts, R.; Stratmann, R. E.; Yazyev, O.; Austin, A. J.; Cammi, R.; Pomelli, C.; Ochterski, J. W.; Ayala, P. Y.; Morokuma, K.; Voth, G. A.; Salvador, P.; Dannenberg, J. J.; Zakrzewski, V. G.; Dapprich, S.; Daniels, A. D.; Strain, M. C.; Farkas, O.; Malick, D. K.; Rabuck, A. D.; Raghavachari, K.; Foresman, J. B.; Ortiz, J. V.; Cui, Q.; Baboul, A. G.; Clifford, S.; Cioslowski, J.; Stefanov, B. B.; Liu, G.; Liashenko, A.; Piskorz, P.; Komaromi, I.; Martin, R. L.; Fox, D. J.; Keith, T.; Al-Laham, M. A.; Peng, C. Y.; Nanayakkara, A.; Challacombe, M.; Gill, P. M. W.; Johnson, B.; Chen, W.; Wong, M. W.; Gonzalez, C.; Pople, J. A. Gaussian 03, Gaussian, Inc., Pittsburgh, PA, 2003., 2003. 